\title{
Study for Implementation of VANET with Transport Layer Protocol
}

\author{
Jaimin C. Shah \\ M.E. Student \\ GCET Engineering \\ College
}

\author{
Kaushika Patel \\ Assistant Professor \\ Electronics Dept \\ BVM Engineering College
}

\author{
Sameer D. Trapasiya \\ Assistant Professor \\ EC Dept. \\ GCET Engineering \\ College
}

\author{
J.M.Rathod, Ph.D \\ Associate Professor \\ Electronics Dept. \\ BVM Engineering College
}

\begin{abstract}
This article includes a comprehensive survey of different routing protocols and mobility models used to develop Vehicular Adhoc Network(VANET). VANETs comprise of vehicle-to-vehicle and vehicle-to-infrastructure communication based on wireless local area network technologies. It is required for safety, and user application. For better visualization, different visualization tools are used to develop VANET architecture. Also different TCP implementation is discussed for study their effect on behaviour of VANET.
\end{abstract}

\section{Keywords}

VANET, NS2, BoonMotion, Routing Protocols, MANET, CWND, RTT

\section{INTRODUCTION}

A Vehicular Ad Hoc Network (VANET) is an established wireless connection between car, functioning of the network closely resembles with the Mobile Ad Hoc Network (MANET). Its high speed mobility change and unpredictable movement characteristics are different for the MANET. In

\begin{abstract}
VANET we used a car as a moving node, each car can receive and transmit the messages through the wireless network, so topologies of the VANETs are randomly change for a small duration of time. In VANET each vehicles communicate with each other via Inter vehicles communication (IVC) as well as roadside unit (RSU), based on it Vehicle to Vehicle (V2V) show in Figure 1 and Vehicle to infrastructure (V2I) show in Figure 2 communication are possible. A special electronic device will be placed inside each vehicle called on-board unit which provides the Ad Hoc network connectivity for the passengers. It will enable the application that provides warning on environmental hazards, as well as traffic and road condition to the vehicle user, thus it is used for safety and comfort for the passengers. The different routing protocols of MANET are very useful to the VANET, the dissimilarity characteristic resulted in frequent loss of connectivity, so it initiated upgradation of the existing routing protocols to develop the VANET. The key parameters which needs to be feed in to this routing protocols is a realistic mobility model which contains linked to speed, road intersection,traffic light effect etc. It's important to know the behavior of transport layer protocols on VA'ET.
\end{abstract}

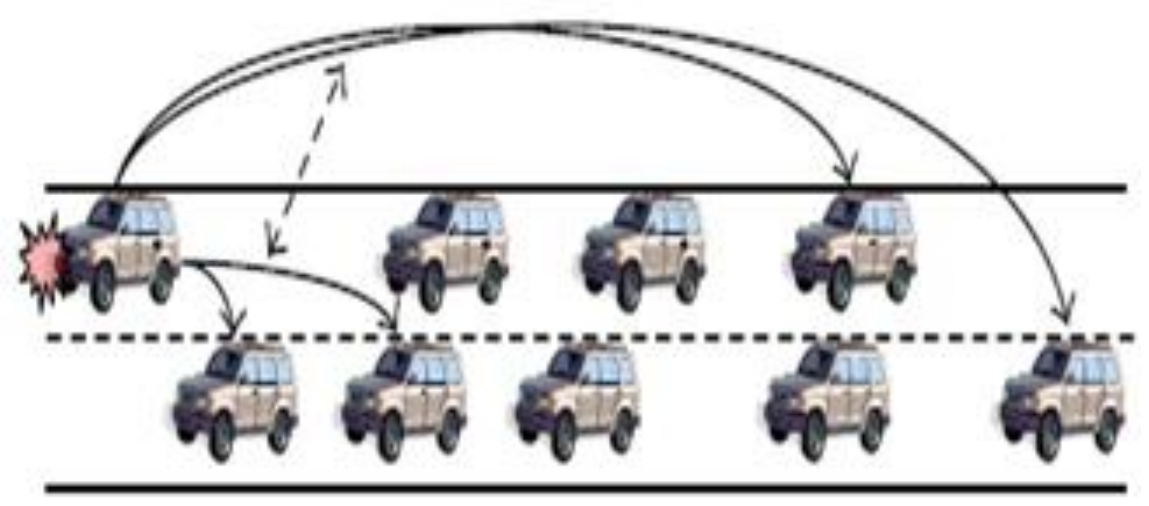

Figure 1 Vehicular to Vehicular network [21] 


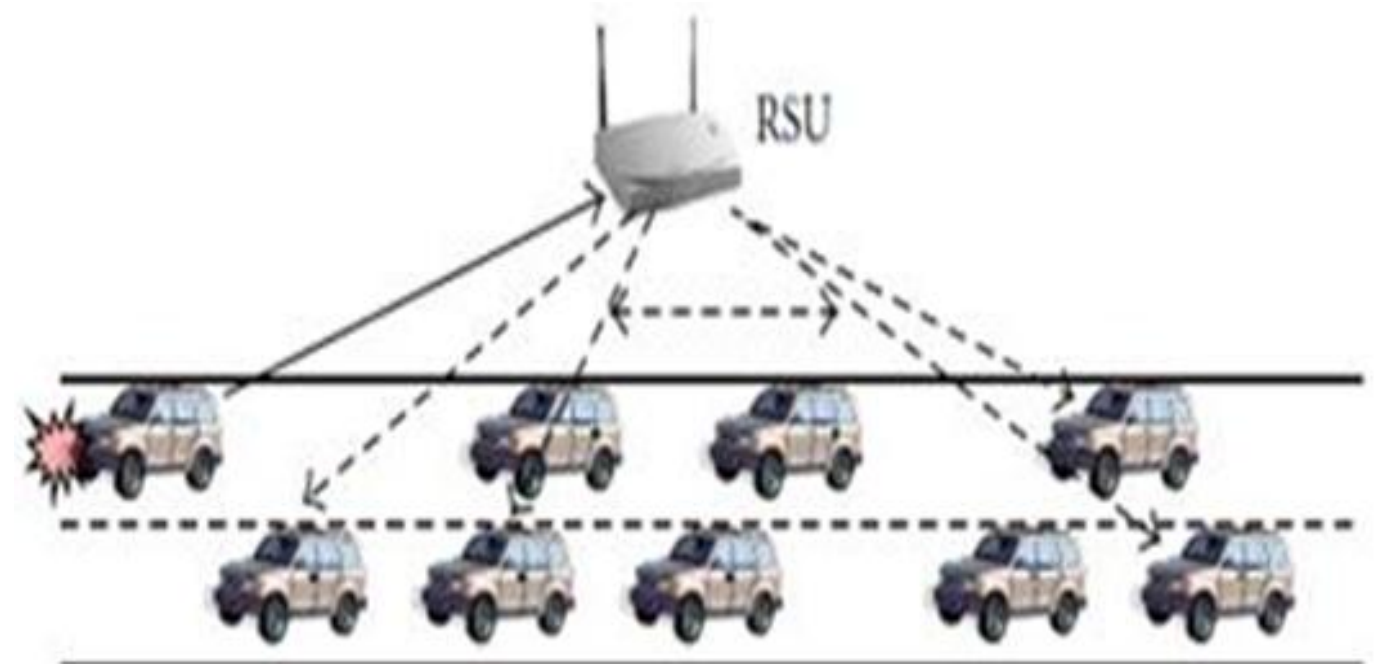

Figure 2 Vehicular to infrastructure communication

The transport layer is responsible for process-to-process delivery of the entire message. Transport layer ensures that the whole message arrives intact and in order. At the transport layer, TCP groups a number of bytes together into a packet called a segment. Following are the protocols at transport layer namely: UDP (User Datagram Protocol), TCP (Transmission Control Protocol), SCTP (Stream Control Transmission Protocol. In VANET has bulk of data for transmission. Different TCP variants are used to avoid the congestion in the dense network condition. To transport the data from the application layer of the source host to that of the destination host transport layer protocol, UDP (User Datagram Protocol) and TCP (Transmission Control Protocol) are used. TCP is used for reliable and connection oriented communication while UDP is used for unreliable and connection less communication. So TCP provide control information related to flow control, error control, congestion control for the messages. It provides the End to End reliable, congestion controlled connection over the Internet. Stream Control Transmission Protocol (SCTP) is a new reliable, message-oriented transport-layer protocol. There are different TCP variants like Tahoe, Reno, New-Reno, SACK, Westwood and WestwoodNR to improve the performance of mattress. Tahoe and Reno are two most common reference implementations of TCP versions. The first modified version in TCP is New-Reno which does not involvesSACK.NewReno overcomes the Reno TCP's performance problems when multiple packet are drop from a window of data [7]. The second version of SACK TCP, is a conservative extension of Reno TCP modified to use the SACK option being proposed in the internet Engineering Task Force (IETF) [1].In wireless network, to optimize the path between source to destination routing protocols are used. Routing protocols are used for finding the shortest path and route the packets in proper order. Next discussion is oriented towards routing protocols on VANET.

\section{ROUTING PROTOCOL IN VANET}

In Ad Hoc network, all the mobile works as a node, the range of the wireless transmission is limited so the communication with the host outside the transmission range host needs to enlist the aid of its nearest possible host for forwarding the packets to the destination. So all the nodes of this network work as a router and takes part in maintenance and discovery of the routing of the nodes in the network. So routing protocols play important role in network.

These routing protocols are mainly divided in to two parts

Table-Drive Routing Protocol: In this type of routing protocol consistent and up-to-date routing information for all nodes are maintained at each node.

On-Demand Routing Protocol: In this type of routing protocol routes are to be created as \& when required, When source want to send the data to the destination, it invokes the routing discovery mechanisms to find the path to the destination.

IVC(Inter-Vehicular Communication) routing protocols are classified in to three major categories likes Broadcast, Multicast and geocastand Unicast [14]. Unicast is subdivided in to the Proactive, Reactive, Prediction Based and Opportunistic based routing protocol as shown in Figure 3.Broadcast based routing scheme is a very basic scheme in which one transmit and all others receive the data so this scheme is one of the solution for high-mobility network, which needs fully distributed network. Geocast based routing protocol [14] is one of the part from the multicast based routing protocol in which one send data and many specified node receive the data from the geographical region. Unicast based routing protocol involves point to point communication. Dynamic nature of VANET can cause serious path disruptions [3]. Therefore, many mechanisms are required to manage the unstable path problem in unicast routing protocols. There are different types of unicast routing protocol. Proactive is a one part of it ,in this type of routing protocol periodically updates and creates the new routes of each pair of vehicles, so its has to handle the problem to find optimum time after new routes have been created. 


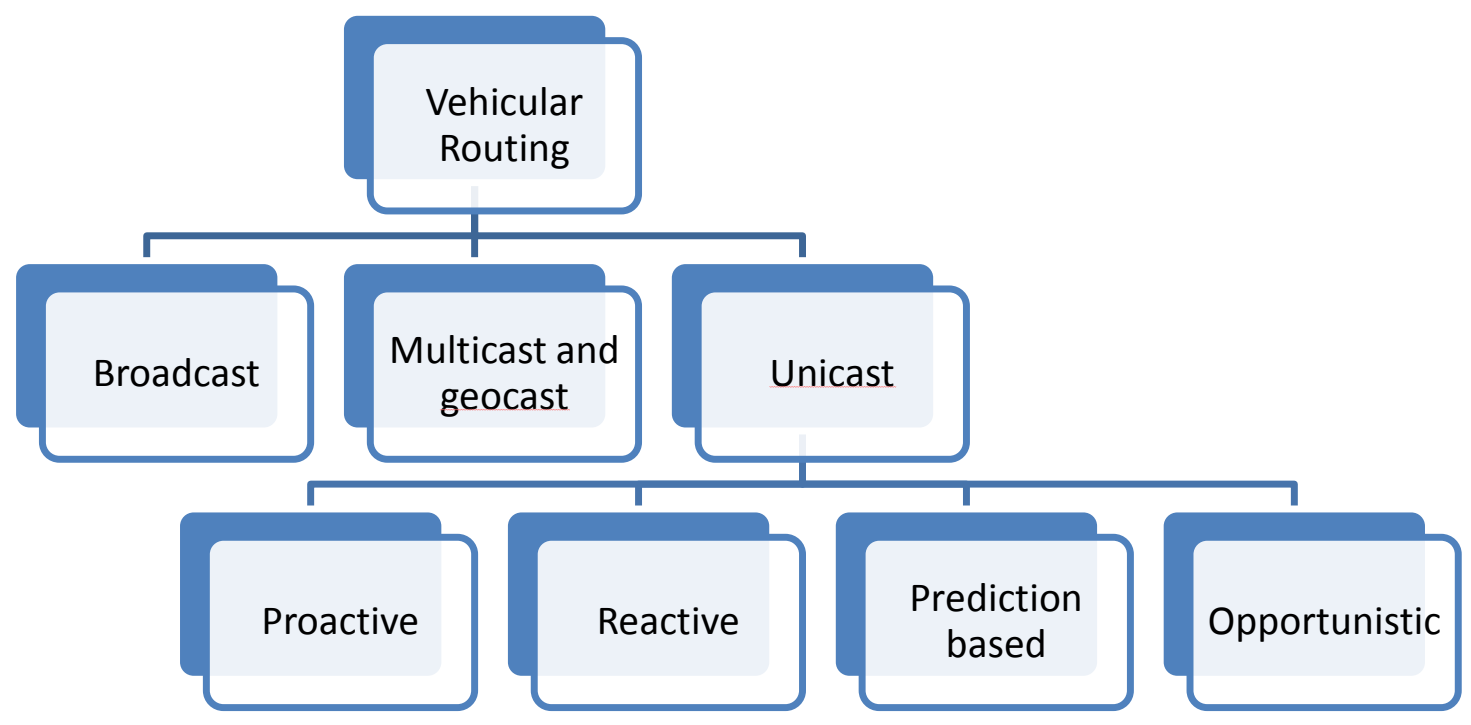

Figure 2 Vehicular Routing Protocols [14]

hort period protocol suffer from the overhead while long time period protocol suffer from frequently route failure. In the reactive type routing protocol the new route is created after breaking the existing route, routing failure probability is very high. Prediction based routing protocol overcomes the problem of both the routing type protocol [14], to predict the probability of routes before the communication is disrupted. Whenever there is no reachable route searched by routing protocol between the pair of vehicle, message would normally be dropped. In an opportunistic manner, routing protocol is a solution to deliver the data even if there is no route between the vehicles. By storing a message until the destination is reached, the messages are then forwarded to the destination with a long delay trade-off. AODV (Ad hoc On Demand Routing Protocol), DSDV (Destination Sequence Distance Vector) and DSR (Dynamic Source Routing) are different routing protocols. Out of these, AODV and DSR are on demand routing protocol and DSDV is a table drive Proactive routing protocol. In DSDV, each node maintains a routing table, which consists of the information related to which node contains the shortest path information to other node in the vehicular network. AODV and DSR are on demand Reactive routing, in which network route only updates when source wants to send a message to the destination vehicle. Mobility Models Mobility models are the movement patterns of nodes communicating in a wireless scenario. In these models, the parameters such as street map structure, obstacle, vehicle density and speed, urban and geographical condition are considered. Mobility models play major role in VANETs. They are classified mostly in two part first one is microscopic and the other one is macroscopic. In macroscopic based mobility models focus is specifically on, motion constraints such as roads, streets, crossroads and traffic light. Also, the generation of vehicular traffic density, traffic flows, and initial vehicle distributions are defined. On the other hand in microscopic approach focuses on the movement of each vehicle and on the vehicle behavior with respect to other vehicles. Different mobility models are available, namely Random Waypoint Mobility Model (RWM), Reference Point Group Mobility Model (RPGM), Manhattan Grid Mobility Model, Random Walk Mobility model, Random Direction Mobility Model, Street Random Waypoint mobility model
(STRAW) and Chain Mobility Model etc. In Random Way point Mobility Model, a random destination point and a uniform speed is attributed to each node. After reaching one destination point, another random destination point is provided. In this model, nodes move randomly in any direction. In RPGM model nodes are moving in the group.For this model to have dynamic groups, when a node comes in the area of another group it changes to this new group with probability. In Manhattan mobility model, nodes are moving in the predefined path only which considers horizontal and vertical move. Different parameters like pause time and minimum or maximum speed can be introduce in this model. In this model grid can be created and normally proposed for the urban areas. It also provides the flexibility for the nodes to change the direction, it imposes the geographic restriction on the node mobility. STRAW is a car following model with US road information to simulate the realistic traffic situation that includes, traffic controls, car interactions, traffic congestion etc in an urban environment. Chain model is not only the model itself but concatenation of different implemented models. With use of Chain model linked with the final position of the model to the initial position of the model. Chain model permit the some specific model, parameters of this models are defined in the Chain model and separated by quotation (" ") .The different common parameters used in these models are number of nodes, max-min speed of the nodes, simulation area, duration maximum pause time. Among them, RWP model is most probably used in the VANET scenario to evaluate the performance of different routing protocol as well as the transport layer different TCP variants.

\section{SIMULATION TOOLS AND SETUP FOR VANET}

Simulation is the imitation of the operation of a real world process or system over time and the simulator is a machine designed tool to provide a realistic imitation of the control and operation of the vehicle, aircraft and other complex system for training purposes. There are a different simulator tools available to develop the different traffic models, mobility models and VANET models. There are major two problems in 
simulation, first one is relative selection [13] of the simulation tool out of dozen of simulation tools available and second one is lack of the common criteria to define simulation experimentation [13]. Basically there are three simulation tools are available, first is mobility simulator, they focuses on generating the mobility traces. Second is network simulator, they perform the data interexchange of the network nodes and third is VANET simulator integrated of the futures of the previous two types. Mobility simulator generates the trace contents the node location and timing details. Open source mobility simulators are SUMO, MOVE, Boon Motion, VanetMobiSim and CityMob and commercial one is PARAMICS [4]. Network simulators evaluate and analyse the different network layer protocols and application layer under different conditions, it is used to develop network layer protocol. There are many network simulators available, out of them some are open source like NS2, GloMoSim, OMNet++ etc and commercial are OPNET and QualNet [4].

\section{Boon Motion}

NS-2

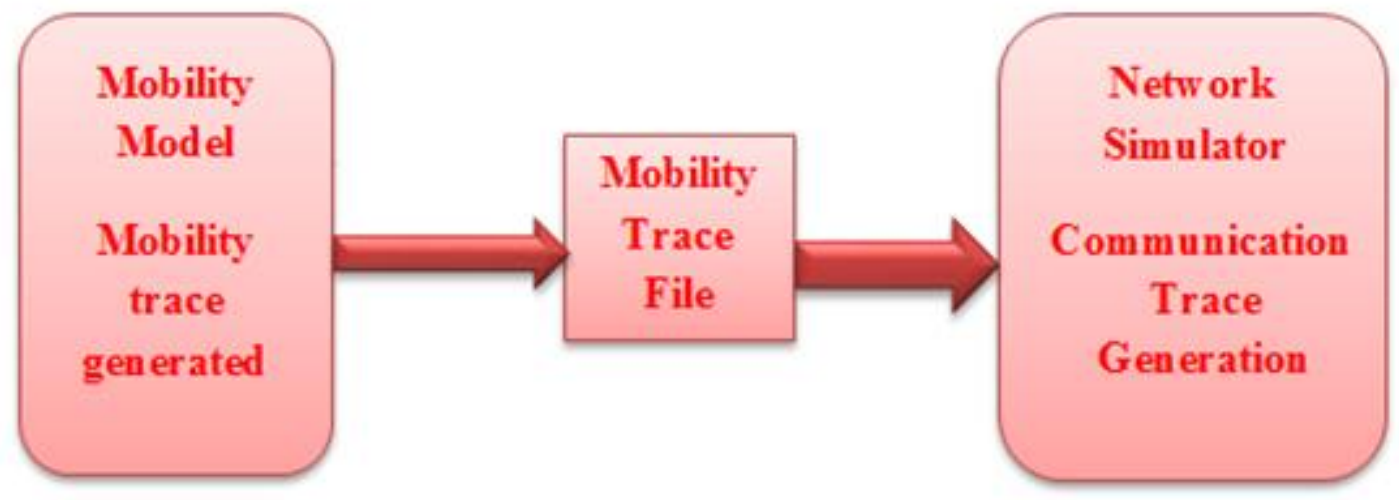

Figure 4 Vehicular Architecture [20]

In VANET simulator the framework of mobility and network simulators is integrated. VANET simulator are TraNS (Traffic and Network Simulation Environment ), NCTUns (National C Sihiao University Network Simulator), iNSpect [13], Groove Net tools are available to simulate the VANET. After studying VANET related papers, it may be seen that different combination of the tools evaluate the different protocol out of them we select the Boon Motion [13] to generate the different mobility models and used NS2 to create network models with use of these two models develop the VANET visualization with use on iNSpect tool. BoonMotion is the java based software which is created and analyses the mobility scenario which can be exported to the network simulators such as ns2, QualNetand others[13]. With the use of it ,develop the different mobility models like RWP mobility model, RPGM mobility model, Manhattan Grid mobility model and Chain mobility model. The traces of BoonMotion saves the movement of nodes based on linear waypoint. So every node contains the one line and this line contains all the waypoints, waypoint give the information about the movement of node changes[13]. In this simulation, time is taken in second at which waypoint is reached by the node, $\mathrm{X}$ and $\mathrm{Y}$ are the coordinate of the position of the waypoint. After generating the mobility parameters, NSFile application is used for converting the mobility scenarios file in to the NS2 compatible format that can be integrated in to the TCL script to start the NS2 simulation.With the use of traffic scenario generated script, generate CBR and TCP traffic between the vehicles nodes called cbrgen.tcl under the NS2. In this define the parameters number of nodes, maximum number of nodes are connected and traffic connection may be TCP or UDP, random seed. For simulation experiment give the mobility trace file and corresponding traffic file to the TCL script in NS2 and generate the trace file. This trace file contains the complete information about the route of the packet flow in to the model, drops and data transmission. With the use of NAM (network Animator) visualize the simulation process which is conducted. The trace file generated from the NS2 simulator was fed to the AWK script which filters out the different performance metrics namely throughput of different tcp over a network, congestion window(CWND) size, Round Trip Time (RTT), Maximum Packet Drop, Number of Retransmissions etc [13]. For better visualization of the VANET, different types of VANET visualization tool are used as discussed above. Out of them iNSpect is a visualization tool which is compatible to the mobility simulator, BoonMotion and network simulator,NS2. The iNSpect program produces a visual display of nodes in wireless scenario ,based on Cartesian $(\mathrm{x}, \mathrm{y})$ coordinate used by ns2. With the use of it visualize the packets transmitted successfully or not, unlike in NAM. The transmissions are displayed with route lines and color coded nodes. Different four color indication in the visualization display, the initiating node of transmission attempt turn blue to indicate it is sending node. The receiving node turns red until the packet is successfully received, then turn green or blue. If the receiving node is the final destination node ,then it turns green otherwise it turns a blue and show that next forwarding transmission attempt, and route line is extended to show the forwarding of the packet[4].

\section{CONCLUSION}

VANETs are a specialized form of MANETs with specific requirements. In this article, we summarized the, transport layer protocols, different routing protocols, mobility models, simulation tools which effectthe performance of VANET. By the use of NS2 large number of nodes can be generated with less simulation time and it is free ware. NS2 supports variety of mobility models for VANET. Boon motion analyses different mobility scenarios which can be exported to NS2. Different routing protocols are used, out of them AODV performs best over wireless channel for different performance metrics like packet delivery ratio and Normalized Routing Load [13]. DSDV has batter performance over AODV for 
End to End delay [13]. For transport layer TCP, WestwoodNR can perform better than the other TCP variants. As it is designed for wireless scenario, it calculates end to end bandwidth after loss of any packet.

\section{FUTURE WORK}

Analysis of TCP WestwoodNR can be done using boon motion and traffic generation model by analysis. Different parameters can be observed and performance can be further improved. This implementation can be applied on real network test bed set.

\section{REFERENCES}

[1] Mr.S.Mohan raj, Mr.S.Kirubakaran, Dr.S.Valarmathy, Mr.E.Praveen Kumar "Performance of TCP Variants in VANET" International Journal of Application or Innovation in Engineering \& Management (IJAIEM) Volume 2, Issue 2, February 2013 ISSN 2319 - 4847.

[2] Mrs.Vaishali D. Khairnar, Dr.KetanKotecha "Simulation-Based Performance Evaluation of Routing Protocols in Vehicular Ad-hoc Network" October 2013, International Journal of Scientific and Research Publications, Volume 3, Issue 10, ISSN 2250-3153

[3] Arvind Kumar Shukla, C.K.Jha, Ph.D., Deepak Sharma " An Estimation of Routing Protocols in Mobility Models Used For Ad Hoc Networks: Simulation Study", 2013, International Conference on Advances in Computer Application (ICACA - 2013).

[4] Patel Kaushika, Rathod Jagdish M., "A Survey on effectiveness of TCP Westwood in mixed wired and wireless network" International journal of scientist \& Engineering Research, Volume 4, Issue 6, june 2013.

[5] Syed A. Hussain and A. Saeed, "An Analysis of Simulators for Vehicular Ad hoc Networks",2013, World Applied Sciences Journal 23 (8): 1044-1048, 2013.

[6] Ms.M.Santhiya1, Mr.M.Sunilkarthick, Ms.M.keerthika "PERFORMANCE OF VARIOUS TCP IN VEHICULAR AD HOC NETWORK BASED ON TIMER MANAGEMENT" DECEMBER 2013, International Journal of Advanced Research in Electrical, Electronics and Instrumentation Engineering, Vol. 2, Issue 12.

[7] HalaEldawIdrisJubara, SharifahHafizah, Syed Ariffin, Shiela N Fisal, NurulMuazzah Abdul Latiff,, Sharifah K Syed Yusof and Rozeha Rashid "Adaptive transport layer protocol for highly dynamic environment", 2012Jubara et al. EURASIP Journal on Wireless Communications and Networking 2012, 2012:229.

[8] Hrituparna Paul, Anish Kumar Saha, ParthaPratim Deb "Comparative Analysis of Different TCP Variants in Mobile Ad-Hoc Network" August 2012. International Journal of Computer Applications (0975 - 8887) Volume 52- No.

[9] Geethu Wilson, Robin Cyriac “ An Enhancement to TCPW BBE by Modifying the Bandwidth Estimation Using Modified EWMA" June 2012, International Journal of Advanced Research in Computer Science and Software Engineering

[10] TajinderKaur, A. K. Verma, " Simulation and Analysis of AODV routing protocol in VANETs" , july 2012, International Journal of Soft Computing and Engineering (IJSCE) ISSN: 2231-2307, Volume-2, Issue-3.
[11] RavinderKaur, Gurpreet Singh Josan "Performance Evaluation Of Congestion Control Tcp Variants In Vanet Using Omnet++" International Journal of Engineering Research and Applications (IJERA), Vol. 2, Issue 5, September- October 2012, pp.1682-1688.

[12] ShimaaHagag, Ayman EI-Sayed(IEEE Senior Member) "Enhanced TCP Westwood Congestion Avoidance Mechanism(TCP WestwoodNew)" International Journal Of Computer Application, Volume-45,Number-5,May2012.

[13] Geethu Wilson, Robin Cyriac"An Enhancement to TCPW BBE by Modifying the Bandwidth Estimation Using Modifieed EWMA" International Journal Of Advanced Research in Computer Science and Software Engineering, June-2012.

[14] SaishreeBharadwaj.P. , Rashmi.S. and Shylaja.B.S., "Performance Evaluation of MANET Based Routing Protocols for VANETs in Urban Scenarios" 2011 International Conference on Network and Electronics Engineering vIPCSIT vol.11 (2011) (C) (2011) IACSIT Press.

[15] ChakkaphongSuthaputchakun and Zhili Sun, "Routing Protocol in Intervehicle Communication Systems: A Survey" IEEE Communication Magazine December 2011.

[16] DiogoAcatauassu, Igor Couto, Patrick Alves, Kelvin Dias, "Performance Evaluation of Inter-Vehicle Communications Based on the Proposed IEEE 802.11p Physical and MAC Layers Specifications" ICN 2011 : The Tenth International Conference on NetworksISBN:978-1-61208-113-7

[17] Mohammad Pasha1 and Mohd Umar Farooq2, "A Priority based Congestion Prevention Technique for Vehicular Ad-Hoc Networks", Proceedings of the 5th National Conference; INDIACom-2011

[18] PrabhakarRanjan1, Kamal Kant Ahirwar, "Comparative Study of VANET and MANET Routing Protocols", 2011, Proc. of the International Conference on Advanced Computing and Communication Technologies (ACCT 2011).

[19] Laila Abdullah Esmeda(1), Mostafa Sami M. Mostafa(1) and Mohamed Mostafa M. Fouad(2) "Performance Evaluation of the IEEE 802.11p Protocol in a VSNET" 2011, Journal of American Science 2011;7(11)

[20] KusumDalal, PrachiChaudhary, Dr.PawanDahiya "Performance Evaluation of TCP and UDP Protocols in VANET Scenarios using NCTUns-6.0 Simulation Tool" December 2011,International Journal of Computer Applications (0975 - 8887) Volume 36- No.6, December 2011.

[21] Arnab Kumar Banik, “ Routing Protocol with prediction based mobility model in Vehicular Ad Hoc Network (VANET) "April, 2010.

[22] M.Kalpana, Dr.T.Purusothaman "Performance Evaluation of Exponential TCP/IP Congestion Control Algorithm" IEEE-International Journal Of Computer Science\&Network Security, March-2009.

[23] ChakkaphongSuthaputchakun, "Priority based intervehicle communication for highway safety messaging 
using IEEE802.11e" International journal of vehicular technology, volume 2009, May 2009.

[24] Yasser toor and Paul mühlethaler, "Vehicle Ad hoc Networks: applications and related technical issues" 3rd quarter 2008, volume 10, no. 3 , IEEE communications.

[25] Samyak Shah, AmitKhandre, Mahesh Shirole and GirishBhole "Performance Evaluation of $\mathrm{Ad} \mathrm{Hoc}$ Routing Protocols Using NS2 Simulation"
[26] Andrew Chen, BehroozKhorashadi, DipakGhosal, ChenNee Chuah, "Impact of Transmission Power on TCP Performance in Vehicular Ad Hoc Networks" ,2007 14244-0860-1/07/25.00 @ 2007 IEEE.

[27] Kazumi KANEKO and Jiro KATTO; “ Reno Friendly TCP Westwood based on Router Buffer Estimation", 2005, proceeding of the joint international Conference on Autonomic and Autonomous Systems and international Conference on Networking and services(ICAC/ICNS) IEEE 0-7695-2450-7-8/05. 